\title{
Metastatic infectious complications in tunneled dialysis catheter-associated infections: a single-center experience
}

\author{
(DErcan Türkmen \\ Ondokuz Mayıs University, Faculty of Medicine, Department of Nephrology, Samsun, Turkey
}

Cite this article as: Türkmen E. Metastatic infectious complications in tunneled dialysis catheter-associated infections: A single-center experience. J Health Sci Med 2022; 5(1): 178-183.

\begin{abstract}
Aim: Although the guidelines recommend arteriovenous fistula (AVF) primarily as vascular access in hemodialysis patients, tunneled catheter (TC) use is gradually increasing. TCs are associated with an increased risk of infection. TC infections can cause many metastatic infectious complications such as infective endocarditis (IE), spondylodiscitis (SpD), and paravertebral abscess. This study aimed to determine the frequency, risk factors, and prognosis of metastatic infectious complications in patients admitted to our hospital with TC infections.

Material and Method: Patients with TCs hospitalized to the Nephrology unit of Ondokuz Mayıs University Hospital between January 1, 2015, and January 1, 2020, with catheter infection, were included in the study. Demographic, clinical, and microbiological information was obtained from the patients' medical records retrospectively. Metastatic infectious complications were defined as IE, SpD, paravertebral or epidural abscess, and septic embolisms in any focus. Binary logistic regression analyzes were used to identify risk factors for metastatic infectious complications.

Results: One hundred and forty-eight catheter episodes were included in the study. Eighty-seven (58.8\%) of the patients were women. Metastatic infectious complications developed in $22(14.9 \%)$ of the patients. Of these, ten patients had IE, ten patients had SpD, and two patients had both IE and SpD. Coagulase-negative staphylococci was obtained as pathogenic microorganism in most cases $(9 / 22,49 \%)$. Patients with infectious complications had higher length of hospital stay [46.5 (10-171) vs 18 (692); $\mathrm{p}<0.001$ ], and higher rates of sepsis ( $50 \%$ vs $16.7 \%$; $\mathrm{p}<0.001$ ), need for intensive care unit ( $36.4 \%$ vs $12.7 \%$; $\mathrm{p}=0.005)$, and death (36.4\% vs $11.9 \% ; \mathrm{p}=0.003)$. In multivariate binary logistic regression analysis, diabetes mellitus (DM) [OR: 7,813; 95\% CI $(2.05-29,783) ; \mathrm{p}=0.003$ ] and catheter duration [OR: $1.002 ; 95 \% \mathrm{CI}(1-1,003) ; \mathrm{p}=0.009]$ were identified as risk factors associated with metastatic infectious complications.

Conclusion: Metastatic infectious complications are associated with significant morbidity and mortality in hemodialysis patients. Long catheter duration and the presence of DM are risk factors for infectious complications. As recommended in international guidelines, minimizing the use of catheters and preventing the development of catheter infection by paying attention to basic hygiene rules, especially in diabetic patients, will help prevent these serious complications.
\end{abstract}

Keywords: Hemodialysis, infective endocarditis, metastatic complications, spondylodiscitis, tunneled catheter

\section{INTRODUCTION}

Among the renal replacement therapy options for endstage renal disease patients, hemodialysis is the most preferred modality both in our country and all over the world (1). Arteriovenous fistula (AVF), synthetic AV grafts, or central venous catheters can be used as vascular access in hemodialysis patients. Although the guidelines recommend AVF primarily as vascular access in hemodialysis patients, central venous tunneled catheter (TC) use gradually increases (2).

Unfortunately, TCs are associated with significant morbidity and mortality in hemodialysis patients. In a meta-analysis, compared with persons with fistulas, those individuals using catheters had higher risks for allcause mortality (risk ratio $=1.53$ ), fatal infections (2.12), and cardiovascular events (1.38) (3). Hospitalization rates for patients with TCs are more than twofold higher than those in patients with AVF (4). Infections are the most common cause of death in dialysis patients after cardiovascular causes (5). Local infections such as tunnel and exit site infections may occur in TCs, and bacteremia can also be seen. A Canadian study showed that during the first six months of dialysis, there is a high rate of bloodstream infection (BSI). In comparison to the $\mathrm{AV}$ 
fistula, survival analysis revealed a relative risk of BSI of 8.49 (95\% CI, 3.03-23.78) for TCs (6). Bacteremia can cause many metastatic infectious complications such as infective endocarditis (IE), spondylodiscitis (SpD), paravertebral abscess. These metastatic complications have also been shown to increase mortality and morbidity (7).

Our knowledge of metastatic complications in tunneled catheter infections is not sufficient. This study aimed to determine the frequency, risk factors, and prognosis of metastatic infectious complications in patients admitted to our hospital with tunneled dialysis catheter infections.

\section{MATERIAL AND METHOD}

The study was carried out with the permission of Ondokuz Mayıs University Clinical Researchs Ethics Committee (Date: 26.08.2021, Decision No: 2021/403). All procedures were carried out in accordance with the ethical rules and the principles of the Declaration of Helsinki.

The study was planned retrospectively. Patients with TCs hospitalized to the Nephrology unit of Ondokuz Mayis University Hospital between January 1, 2015, and January 1, 2020, with catheter infection were included in the study. Patients with a temporary catheter and individuals under the age of 18 were excluded from the study.

Catheter-associated infection was defined as positive blood cultures or catheter cultures and no source of infection other than the catheter.

Demographic, clinical, and microbiological information was obtained from the patients' medical records. Data included age, gender, etiology of chronic kidney disease, duration of dialysis, clinical symptoms, and inflammation parameters [C-reactive protein (CRP), procalcitonin, erythrocyte sedimentation rate (ESR), white blood cell count] and biochemical parameters. Microbiological data included the type of microorganism and antimicrobial treatments. In addition, the treatments applied for the infected catheter (withdraw or exchange of catheter, transition to AVF, AV graft, or peritoneal dialysis) were also determined.

Metastatic infectious complications were defined as IE, SpD, paravertebral or epidural abscess, and septic embolisms in any focus. When vegetation was detected by echocardiography, the presence of IE was clinically confirmed and used in the analyses. The diagnoses of $\mathrm{SpD}$, paravertebral and epidural abscess, and septic embolism were accepted as definitive diagnose when supported by clinical findings and characteristic changes detected in radiological evaluation.
When there was more than one episode of catheter infection in the same patient, each episode was considered as a separate patient in the analyses. However, since the primary endpoint was the presence of metastatic complication if a patient with metastatic infection continued with a catheter and re-infection developed, these episodes were not included in the analysis.

\section{Statistical Analysis}

All analyzes were conducted using the Statistical Package for the Social Sciences for Windows, version 25 (SPSS Inc., Chicago, IL, USA). Normality distribution was evaluated with the Shapiro-Wilk and KolmogorovSmirnov tests. The continuous variables were expressed as mean \pm standard deviation in the variables showing normal distribution and median (minimum-maximum) in those that did not show normal distribution. Categorical variables are expressed as percentages. Independent Samples T-test was used to compare non-dependent nonparametric variables in those with normal distribution, and Mann Whitney $U$ test was used in those without normal distribution. The comparison of parametric variables was made with the Pearson Chi-Square test. Binary logistic regression analyzes were used to identify risk factors for metastatic infectious complications. P values $<0,05$ were considered statistically significant.

\section{RESULTS}

One hundred and forty-eight catheter episodes were included in the study. The median and mean ages of the patients were $62.5(20-80)$ and $58.26 \pm 15.61$ years, respectively. Eighty-seven (58.8\%) of the patients were women. Diabetic nephropathy was the most common etiological cause of chronic kidney disease in 57 $(38.5 \%)$ of the patients, while urological causes were the second most frequent. While $105(70.9 \%)$ patients had hypertension, 21 (14.2\%) patients had malignancy. The majority of patients (67.6\%) had a right jugular catheter. The left jugular catheter was present in $41(27.7 \%)$ patients, whereas the femoral catheter was present in only 7 (4.8\%) patients. Fever was the most common complaint (85.1\%) at admission. Eight (5.4\%) of the patients had low back pain or neurological symptoms. Among the microbiological agents, the most common causative agent was staphylococci (53.4\%), gram-negative bacilli were the second most common (25\%). The culture was negative in $12.8 \%$ of catheter infection episodes.

Metastatic infectious complications developed in 22 (14.9\%) of the patients. Of these, ten patients had IE, ten patients had SpD, and two patients had both IE and SpD. Paravertebral abscess accompanied in 7 (63.6\%) of the patients with $\mathrm{SpD}$. Mitral valve vegetation was detected in most patients with IE $(7 / 11 ; 63.6 \%)$. While the 
causative agent was coagulase-negative staphylococcus $(9 / 22,49 \%)$ in most of the patients with infectious complications, $S$. aureus was the causative agent in $6(27.3 \%)$ patients. While $73(49.3 \%)$ of the catheter infections were treated without catheter withdrawal or exchange, the catheter was exchanged in 59 (39.9\%) episodes. Transition to AV fistula or peritoneal dialysis was performed in $16(10.8 \%)$ episodes. Sepsis was observed in $32(21.6 \%)$ episodes, and the need for intensive care developed in 24 (16.2\%). In addition, the mortality rate was $15.5 \%(23 / 148)$.

Compared to those without metastatic infection, patients with infectious complications had higher ESR and CRP values, a higher incidence of diabetes mellitus (DM), longer catheter duration and length of hospital stay, and higher rates of sepsis, need for intensive care unit, and death. However, albumin levels were significantly low (Table 1). In multivariate binary logistic regression analysis, DM [OR: 7.813; 95\% CI (2.05 - 29.783); $\mathrm{p}=0.003$ ] and catheter duration [OR: 1.002; 95\% CI (1$1.003) ; \mathrm{p}=0.009$ ] were identified as risk factors associated with metastatic infectious complications (Table 2). While $9(40.9 \%)$ patients with metastatic infection were treated without catheter withdrawal or exchange, catheter exchange was performed in 6 patients $(27.3 \%)$ with IE, 4 patients (18.2\%) with SpD and one patient (4.5\%) with IE and $\mathrm{SpD}$. One patient was transitioned to AVF, and one patient to peritoneal dialysis.

\section{Table 1. Comparison of demographic, clinical and laboratory characteristics of patients according to metastatic infectious complication}

\begin{tabular}{|c|c|c|c|c|}
\hline & \multicolumn{2}{|c|}{ Metastatic infectious complication } & \multirow{2}{*}{$\mathbf{t}$} & \multirow{2}{*}{$\mathbf{p}$} \\
\hline & No $(n=126)$ & Yes $(n=22)$ & & \\
\hline Age, year & $61(20-80)$ & $63.5(28-80)$ & 1182.5 & $0.272^{\mathrm{b}}$ \\
\hline $\mathrm{ESR}, \mathrm{mm} / \mathrm{h}$ & $79.88 \pm 32.39$ & $96.33 \pm 31.63$ & -2.148 & $0.034^{\mathrm{a}}$ \\
\hline $\mathrm{CRP}, \mathrm{mg} / \mathrm{L}$ & $68(0.3-482)$ & $153.5(17-306)$ & 872 & $0.006^{\mathrm{b}}$ \\
\hline Procalcitonin, $\mathrm{ng} / \mathrm{mL}$ & $8.58(0.2-234)$ & $6.65(0.31-100)$ & 1227 & $0.944^{\mathrm{b}}$ \\
\hline ALT, U/L & $9(2-128)$ & $9(2-344)$ & 1378.5 & $0.968^{\mathrm{b}}$ \\
\hline AST, U/L & $15(4-171)$ & $13(5-328)$ & 1354.5 & $0.865^{\mathrm{b}}$ \\
\hline Albumin, gr/dL & $3.34 \pm 0.73$ & $2.91 \pm 0.57$ & 2.634 & $0.009^{\mathrm{a}}$ \\
\hline $\mathrm{WBC}, \mu \mathrm{L}$ & $9660(197.1-31700)$ & $10550(1050-39460)$ & 1169 & $0.242^{\mathrm{b}}$ \\
\hline Hemoglobin, gr/dL & $9.82 \pm 1.79$ & $9.41 \pm 1.55$ & 1.020 & $0.309^{\mathrm{a}}$ \\
\hline Platelet, $\times 10^{3} / \mu \mathrm{L}$ & $182(16.1-602)$ & $167.5(37-540)$ & 1185 & $0.279^{\mathrm{b}}$ \\
\hline Length of hospital stay, day & $18(6-92)$ & $46.5(10-171)$ & 572 & $<0.001^{\mathrm{b}}$ \\
\hline Catheter duration, day & $202(5-1880)$ & $387.5(18-2706)$ & 975.5 & $0.027^{\mathrm{b}}$ \\
\hline Dialysis vintage, month & $15(1-320)$ & $13.5(1-146)$ & 1385 & $0.996^{\mathrm{b}}$ \\
\hline Gender (Female), n (\%) & $76(60.3)$ & $11(50)$ & 0.823 & 0.364 \\
\hline Diabetes mellitus, n (\%) & $43(34.1)$ & $14(63.6)$ & 6.887 & 0.009 \\
\hline Hypertension, n (\%) & $86(68.3)$ & $19(86.4)$ & 2.98 & 0.084 \\
\hline Cardiovascular disease, $\mathrm{n}(\%)$ & $50(39.7)$ & $11(50)$ & 0.823 & 0.364 \\
\hline Malignancy, n (\%) & $17(13.5)$ & $4(18.2)$ & 0.338 & 0.561 \\
\hline Microbiological agent, n (\%) & & & 5.503 & 0.358 \\
\hline S. aureus & $24(19)$ & $6(27.3)$ & & \\
\hline CNS & $41(32.5)$ & $9(40.9)$ & & \\
\hline Gram-negative bacilli & $33(26.2)$ & $4(18.2$ & & \\
\hline Sepsis, n (\%) & $21(16.7)$ & $11(50)$ & 12.28 & $<0.001$ \\
\hline ICU need, n (\%) & $16(12.7)$ & $8(36.4)$ & 7.72 & 0.005 \\
\hline Death, n (\%) & $15(11.9)$ & $8(36.4)$ & 8.537 & 0.003 \\
\hline
\end{tabular}

\begin{tabular}{|c|c|c|c|c|}
\hline \multirow{2}{*}{ Variables } & \multicolumn{2}{|c|}{ Univariate } & \multicolumn{2}{|c|}{ Multivariate } \\
\hline & OR (\%95 CI) & $\mathbf{p}$ & OR (\%95 CI) & p \\
\hline Age & $1.023(0.99-1.057)$ & 0.180 & $1.001(0.954-1.051)$ & 0.955 \\
\hline Gender (Male) & $1.52(0.613-3.771)$ & 0.366 & $2.378(0.743-7.614)$ & 0.145 \\
\hline Diabetes mellitus (Yes) & $3.378(1.315-8.678)$ & 0.011 & $7.813(2.05-29.783)$ & 0.003 \\
\hline Hypertension (Yes) & $2.946(0.824-10.533)$ & 0.097 & $1.783(0.37-8.586)$ & 0.471 \\
\hline Cardiovascular disease (Yes) & $1.520(0.613-3.771)$ & 0.366 & $1.132(0.343-3.734)$ & 0.838 \\
\hline Malignancy (Yes) & $1.425(0.43-4.721)$ & 0.562 & $3.185(0.716-14.164)$ & 0.128 \\
\hline \multicolumn{5}{|l|}{ Catheter localization } \\
\hline Right jugular & \multicolumn{2}{|c|}{ Reference } & \multicolumn{2}{|c|}{ Reference } \\
\hline Left jugular/Femoral & $0.967(0.366-2.556)$ & 0.947 & $0.852(0.237-3.07)$ & 0.807 \\
\hline Hemoglobin & $0.869(0.663-1.138)$ & 0.307 & $0.873(0.643-1.185)$ & 0.384 \\
\hline S. aureus infection (Yes) & $1.875(0.657-5.351)$ & 0.240 & $3.125(0.917-10.643)$ & 0.068 \\
\hline Catheter duration & $1.001(1.000-1.002)$ & 0.014 & $1.002(1-1.003)$ & 0.009 \\
\hline
\end{tabular}




\section{DISCUSSION}

Our study found the incidence of metastatic infectious complications in tunneled dialysis catheter infections to be $14.9 \%$. We found long catheter time and the presence of DM to be risk factors for the development of metastatic infectious complications. We also showed that the length of hospital stay, sepsis, and mortality rates are higher in cases with complications.

International guidelines recommend AVF for longterm vascular access in hemodialysis patients. However, tunneled catheters are frequently used for reasons such as patient selection, initiation of dialysis under emergency conditions, and inability to open AVF to the patient. In a recent observational cohort study, the risk of developing catheter-related complications at one year was $30 \%$, and the risk of bacteremia was $9 \%$. In the second year, bacteremia increased to $11 \%$. In the same study, bacteremia was found to be the most common cause of hospitalizations (73\%) (8). A large meta-analysis showed that catheter use causes a 2-4 fold increase in the risk of fatal and non-fatal infections, with increased mortality (53\%) and hospitalization (68\%) compared to AVF (3).

Chronic kidney disease, hemodialysis, and especially a catheter as vascular access pose a risk for infectious complications such as IE and SpD (9-12). However, data on the frequency of infectious complications after bacteremia in hemodialysis patients are scarce. Mokrzycki et al. (13) found an infectious complication rate of $7 \%$ in their study. In our study, this rate was found to be $14.9 \%$. However, since there is no other data in the literature, the necessity of new studies on its actual frequency is obvious.

On the other hand, although it is known that catheter use in hemodialysis patients increases the risk of infection, our information on the factors affecting complications is not sufficient. Mokrzycki et al. (13) reported that the only variable in a multivariate analysis that was significantly associated with the development of an infectious complication was infection with $S$. aureus. Similarly, the most common causative organism in developing IE or $\mathrm{SpD}$ in dialysis patients is $S$. aureus $(10,14,15)$. Parallel to these, complications are common in $S$. aureus bacteremia developing in hemodialysis patients, and it has been shown that $17 \%$ of the patients develop IE, and $5.7 \%$ of them develop osteomyelitis (16). In our study, $S$. aureus was found to be the causative agent in 6 of the patients who developed complications, but it was not detected as a risk factor for the development of complications in the multivariate analysis. Most of our patients were found to be coagulase-negative staphylococcal factors. In other studies in the literature, similar to ours, it is noteworthy that staphylococci are the most common factor in cases that develop both IE and SpD.
In our study, the presence of DM and the catheter duration were determined as risk factors for complications. Diabetes creates susceptibility to infections by affecting both cellular and humoral immunity (17). In a study conducted on a large group of hemodialysis patients with catheters, DM increased the risk of developing catheter infection 2.37 times (18). On the contrary, some studies show that DM does not cause an increase in the risk of infection $(8,19)$. However, there is no previous study showing that DM poses a risk for metastatic infectious complications. In our study, DM was found to be a severe risk factor for the development of metastatic infectious complications (OR:7.8). It is known that there is a relationship between the duration of the catheter and the development of catheter-related infections. Lemaire et al. (18) found that a catheter duration of $>90$ days increased the risk of infection. Shingarev et al. (20) determined that there was a serious increase in the rate of infected patients with the increase in the duration of the catheter $\left(35 \%\right.$ at the $3^{\text {rd }}$ month vs. $79 \%$ at the $12^{\text {th }}$ month).

Studies show that the catheter location (left internal jugular vein or femoral veins) is associated with an increased risk of infection $(21,22)$. However, on the contrary, it has been shown in many studies that catheter location is not one of the risk factors associated with the development of infection $(23,24)$. It has even been found that infection rates in femoral tunneled catheters are similar to those in jugular catheters $(19,25)$. It is not known whether the catheter location poses a risk for metastatic infectious complications. However, in our study, we did not find a relationship between the catheter location and the development of complications.

Our study found both the length of hospital stay and mortality rate significantly higher in patients with complications than those without complications. In addition, the development of sepsis and the need for intensive care were higher in these patients. The median length of stay was 46.5 days, and the mortality was $36.4 \%$ in patients with complications. Considering that patients should receive intravenous treatment for an average of 6 weeks, the length of hospital stay was prolonged. Similar or even longer lengths of hospital stay are observed in other studies in the literature $(14,15)$. The length of stay in hospital also differs significantly higher when compared with the non-dialysis population (26). Severe infections in patients with complications have led to sepsis, in parallel, to an increase in the need for intensive care. In addition, disease severity is also associated with increased mortality rates. Although the mortality rate was relatively high in our study, similarly, the mortality rate in other studies in the literature ranged between $20 \%$ and $46 \%(14,15,26-28)$. 
Our study has some limitations. First of all, the most important limitations are that it is retrospective, and it was performed only in patients hospitalized in a singlecenter or even only in the nephrology unit. In addition, the fact that our patients were only patients with TCs may not reflect the incidence of infectious metastatic complications in all hemodialysis patients. However, since TCs are be preferred in the long-term treatment of patients, we think that it is more accurate to determine the frequency in patients with TCs.

\section{CONCLUSION}

Our study showed that metastatic infectious complications (infective endocarditis and spondylodiscitis) are common in TCs related infections. The length of hospital stay and mortality rates increase in patients with metastatic infectious complications. Long catheter duration and presence of DM were identified as risk factors for metastatic infectious complications. As recommended in international guidelines, minimizing the use of catheters and preventing catheter infection by paying attention to basic hygiene rules, especially in diabetic patients, will help prevent these serious complications

\section{ETHICAL DECLARATIONS}

Ethics Committee Approval: The study was carried out with the permission of Ondokuz Mayıs University Clinical Research Ethics Committee (Date: 26.08.2021, Decision No: 2021/403).

Informed Consent: Because the study was designed retrospectively, no written informed consent form was obtained from patients.

Referee Evaluation Process: Externally peer-reviewed.

Conflict of Interest Statement: The author has no conflicts of interest to declare.

Financial Disclosure: The author declared that this study has received no financial support.

Author Contributions: The author declare that they have all participated in the design, execution, and analysis of the paper, and that they have approved the final version.

Note: Part or all of the manuscript is not published elsewhere and is not in the process of being evaluated in another journal at the same time. But it was presented as an oral presentation at '23. Ulusal Hipertansiyon and Böbrek hastalıkları Kongresi- 17-22 Eylül 2021, Girne-Kıbrıs'

\section{REFERENCES}

1. Türk Nefroloji Derneği Yayınları. Türkiye'de Nefroloji, Diyaliz ve Transplantasyon. Ankara, 2021. https: //nefroloji.org.tr/uploads/ folders/file/REGISTRY_2020.pdf

2. Murea M, Geary RL, Davis RP, Moossavi S. Vascular access for hemodialysis: A perpetual challenge. Semin Dial 2019; 32: 527-34.
3. Ravani P, Palmer SC, Oliver MJ, et al. Associations between hemodialysis access type and clinical outcomes: a systematic review. J Am Soc Nephrol 2013; 24: 465-73.

4. Klevens RM, Edwards JR, Andrus ML, Peterson KD, Dudeck MA, Horan TC. NHSN Participants in Outpatient Dialysis Surveillance. Dialysis Surveillance Report: National Healthcare Safety Network (NHSN)-data summary for 2006. Semin Dial 2008; 21: 24-8.

5. Lukowsky LR, Kheifets L, Arah OA, Nissenson AR, KalantarZadeh K. Patterns and predictors of early mortality in incident hemodialysis patients: new insights. Am J Nephrol 2012; 35: 54858 .

6. Taylor G, Gravel D, Johnston L, Embil J, Holton D, Paton S. Canadian Nosocomial Infection Surveillance Program; Canadian Hospital Epidemiology Committee. Incidence of bloodstream infection in multicenter inception cohorts of hemodialysis patients. Am J Infect Control 2004; 32: 155-60.

7. Lewis SS, Sexton DJ. Metastatic complications of bloodstream infections in hemodialysis patients. Semin Dial 2013; 26: 47-53.

8. Poinen K, Quinn RR, Clarke A, et al. Complications drom tunneled hemodialysis catheters: a Canadian observational cohort study. Am J Kidney Dis 2019; 73: 467-75.

9. Abbott KC, Agodoa LY. Hospitalizations for bacterial endocarditis after initiation of chronic dialysis in the United States. Nephron 2002; 91: 203-9.

10. Mylona E, Samarkos M, Kakalou E, Fanourgiakis P, Skoutelis A. Pyogenic vertebral osteomyelitis: a systematic review of clinical characteristics. Semin Arthritis Rheum 2009; 39: 10-7.

11. Abid S, DE Silva S, Warwicker P, Farrington K. Infective spondylodiscitis in patients on high-flux hemodialysis and online hemodiafiltration. Hemodial Int 2008; 12: 463-70.

12. Priest DH, Peacock JE Jr. Hematogenous vertebral osteomyelitis due to Staphylococcus aureus in the adult: clinical features and therapeutic outcomes. South Med J 2005; 98: 854-62.

13. Mokrzycki MH, Zhang M, Cohen H, Golestaneh L, Laut JM, Rosenberg SO. Tunnelled haemodialysis catheter bacteraemia: risk factors for bacteraemia recurrence, infectious complications and mortality. Nephrol Dial Transplant 2006; 21: 1024-31.

14. Madhavan K, Chieng LO, Armstrong VL, Wang MY. Spondylodiscitis in end-stage renal disease: a systematic review. J Neurosurg Spine 2019; 15: 1-9.

15. Rocha A, Castro R, Santos J. Endocarditis and spondylodiscitis associated with tunneled cuffed hemodialysis catheters: hospitalizations with poor outcomes. Int J Artif Organs 2015; 38: 173-7.

16.Engemann JJ, Friedman JY, Reed SD, Griffiths RI, Szczech LA, Kaye KS, et al. Clinical outcomes and costs due to Staphylococcus aureus bacteremia among patients receiving long-term hemodialysis. Infect Control Hosp Epidemiol 2005; 26: 534-9.

17. Koh GC, Peacock SI, van der Poll T, Wiersinga WJ. The impact of diabetes on the pathogenesis of sepsis. Eur J Clin Microbiol Infect Dis 2012; 31: 379-88.

18. Lemaire X, Morena M, Leray-Moragués $\mathrm{H}$, et al. analysis of risk factors for catheter-related bacteremia in 2000 permanent dual catheters for hemodialysis. Blood Purif 2009; 28: 21-8.

19. Guillermo-Corpus G, Ramos-Gordillo JM, Peña-Rodríguez JC. Survival and clinical outcomes of tunneled central jugular and femoral catheters in prevalent hemodialysis patients. Blood Purif 2019; 47: 132-9.

20. Shingarev R, Barker-Finkel J, Allon M. Natural history of tunneled dialysis catheters placed for hemodialysis initiation. J Vasc Interv Radiol 2013; 24: 1289-94.

21.Engstrom BI, Horvath JJ, Stewart JK, et al. Tunneled internal jugular hemodialysis catheters: impact of laterality and tip position on catheter dysfunction and infection rates. J Vasc Interv Radiol 2013; 24: 1295-302. 
22.Zanoni F, Pavone L, Binda V, et al. Catheter-related bloodstream infections in a nephrology unit: Analysis of patient- and catheterassociated risk factors. J Vasc Access 2021; 22: 337-43.

23. Coker MA, Black JR, Li Y, et al. An analysis of potential predictors of tunneled hemodialysis catheter infection or dysfunction. J Vasc Access 2019; 20: 380-5.

24.Donati G, Spazzoli A, Croci Chiocchini AL, et al. Bloodstream infections and patient survival with tunneled-cuffed catheters for hemodialysis: A single-center observational study. Int J Artif Organs 2020; 43: 767-73.

25.Sepas HN, Negahi A, Mousavie SH, Vosough F, Farazmand B. Patency and outcomes of tunneled hemodialysis catheter via femoral versus jugular vein access. J Adv Pharm Technol Res 2019; 10: 81-4.

26. Kuo G, Sun WC, Lu YA, et al. Chronic dialysis patients with infectious spondylodiscitis have poorer outcomes than nondialysis populations. Ther Clin Risk Manag 2018; 14: 257-63.

27. Kamalakannan D, Pai RM, Johnson LB, Gardin JM, Saravolatz LD. Epidemiology and clinical outcomes of infective endocarditis in hemodialysis patients. Ann Thorac Surg 2007; 83: 2081-6.

28. Rekik S, Trabelsi I, Hentati M, et al. Infective endocarditis in hemodialysis patients: clinical features, echocardiographic data and outcome: a 10-year descriptive analysis. Clin Exp Nephrol 2009; $13: 350-4$ 